\title{
A New Code for Comprehensive Self-Consistent Modelling of Planetary Nebula Luminescence
}

\author{
V.V. Golovaty ${ }^{1}$, Yu.F. Malkov ${ }^{2}$ and O.V. Rokach ${ }^{1}$ \\ ${ }^{1}$ Astronomical Observatory of Lviv State University, Lviv, Ukraine; \\ ${ }^{2}$ Crimean Astrophysical Observatory, Nauchny, Crimea, Ukraine
}

A new code PAN (Photoionized Axially-symmetrical Nebula) for the self-consistent modelling of a cylindrical gaseous nebula photoionized by a central source of ionizing radiation is developed. All of the processes which play an important role in the ionization and thermal equilibrium of the nebular gas are included in the code.

Distinctive features of the code are the broken analytical solution of the transfer equation for the direct ionizing radiation, complete account for the diffuse ionizing radiation, the possibility to calculate models with realistic central source spectrum and realistic density distribution in the envelope, including multi-shell envelopes and clumped ones. Userfriendly internal structure of both code and atomic data files allows to modify the list of available elementary processes as well as the set of selected energy levels for each ion easily. Different representations of atomic data (tables, various analytical approximations, constants etc.) can be used simultaneously.

The code can be applied for the calculation of photoionization models of planetary nebulae, H II regions, supernova remnants and narrow-line regions in active galactic nuclei. 